\title{
Die Erzeugung teerfreien Gases in einem Gleichstrom-Festbett-Vergaser *
}

\author{
M. J. Groeneveld, P. E. Gellings und J. J. Hos \\ Energy Equipment Engineering B.V., Oldenzad, Niederlande
}

In der vorliegenden Arbeit werden die Grundbegriffe, der Maschnenbau und die Praxis der Gleichstrom-Festbett-Vergasung beschrieben. Für das technische Verfahren ist es wichtig. den Vergaser so zu entwerfen, daßer teerfreies Gas produziert. Basierend auf den Eigenschaften des Brennstoffs, an die der Glutherd entsprechend angepaßt werden muß, wird im Falle höherer Kapazitäten und feinen Brennstoffs ein kreisrunder Brennkammer-Hals angewandt. Es werden Modelle zur Berechnung der maximalen Kapazität unter Berücksichtigung der Teerproduktion und für den Entwurf der Reduktionszone angegeben. Für den Maschinenbau wird über die Brennstollbehandlung. die Auswahl des Materials. die Bescitigung der Asche, über Behandlung und Gebrauch des Gases und die Sicherheitsaspekte berichtet. Der Stand der praktischen Anwendung von Vergasern wird dargestellt. Aus der wirtschaftlichen Bewertung folgt, dál die Vergasung eine rentable Möglichkeit sowohl für die direkıc Beheizung als auch für die Wärme/Kraft-Kopplung ist.

\section{Production of a tar-free gas in an annular co-current moving bed gasifier}

This paper discusses the fundamentals, mechanical engineering and commercialisation of co-current moving bed gasifiers. The process engineering aspect stresses that gasiliers can be designed to obtain tar-free product gas. Based on the fuel properties, the fire hearth has to be adjusted, with an annular throat concept applied in the case of lirger capacities and fine fuel. Models are presented for maximum capacity calculations of gasiliers with respect to tar production and for reduction zone design. From a mechanical engineering aspect fuel handing, materials selection, ash removal, gas treatment and utilizàtion as well as safety risks are discussed. The present state of gasifier commercialization is given. The economic evaluation leads to the conclusion that gasification is a viable option for direct heating and co-generation.

\section{Einleitung}

Gleichstrom-Festbett-Vergaser werden seit Anfang dieses Jahrhunderts zur Vergasung von Biomasse eingeselzt und auch heute noch sind diese Reaktor-Typen im Vergleich mit anderen Vergasertypen durchaus interessant. Ihre Hauptvorteile sind darin zu sehen, daß der Reaktor einfach in Handhabung und Kontrolle ist und daß ein teerfreies Gas hergestellt wird, das zur Kraft- und/oder Wärmeerzeugung benutzt werden kann.

\footnotetext{
* Die Untersuchungen wurden größtenteils an oder in Zusammenarbeit mit der Technischen Universität Twente, Enschede, Niederlande, durchgeführt. Dic augenblickliche Entwicklungsarbeit wird von der Maschinefabriek Oldenzaal und dem niederländischen Wirtschaftsministerium finanziert.
}

Die klassischen Konstruktionen sind jedoch für die Vergasung von Brennstoffen mit kleiner Teilchengröße oder hohem Aschengehalt ungeeignet und sie sind nur schwer zu vergrößern. Das derzeitige Wiederaufleben des Interesses an der Biomasse-Vergasung macht die Nutzung von Brennstoffen. variierend in Teilchengröße. Feuchtigkeit und Aschengehait etc. vorstellbar. Zur Vergasung dieser Brennstoffe muß für die Vergaser-Konstruktion die Analyse der physikalischen und chemischen Vorgänge während der Vergasung berücksichtigt werden. Die Analyse führt zu dem BrennkammerHals-Konzept, das die Herstellung teerfrei arbeitender Vergaser für viele Brennstoffarten mit Kapazitäten von $10 \ldots 1000 \mathrm{~kg} / \mathrm{h}$ ermöglicht.

Die Vergasung selbst ist ein komplexer Prozess. Der feste Brennstoff trocknet, verschwelt und reagiert mit $\mathrm{O}_{2}, \mathrm{CO}_{2}$ und $\mathrm{H}_{2} \mathrm{O}$ wie nachstehend in vereinfachter Form dargestellt:

$$
\begin{aligned}
\mathrm{C}+\mathrm{O}_{2} & =\mathrm{CO}_{2} \\
\mathrm{C}+\mathrm{CO}_{2} & =2 \mathrm{CO} \\
\mathrm{C}+\mathrm{H}_{2} \mathrm{O} & =\mathrm{CO}+\mathrm{H}_{2} \\
\mathrm{CO}+\mathrm{H}_{2} \mathrm{O} & =\mathrm{CO}_{2}+\mathrm{H}_{2}
\end{aligned}
$$

Im Vergaser (Bild 1) findet man dic einzelnen Schritte des Prozesses in verschiedenen Zonen, die sich normalerweise überlappen. Der Festbrennstoff wird oben in den Vergaser eingeführt und strömt nach unten, wobei er zunächst in Holzkohle und schließlich in Asche umgewandelt wird.

Das Trocknen und die Schwelgasbildung finden während des Wärmeaustausches mit der Oxidationszone in der Nähe des Lufteinlasses statt. In der Oxidationszone haben die Re-

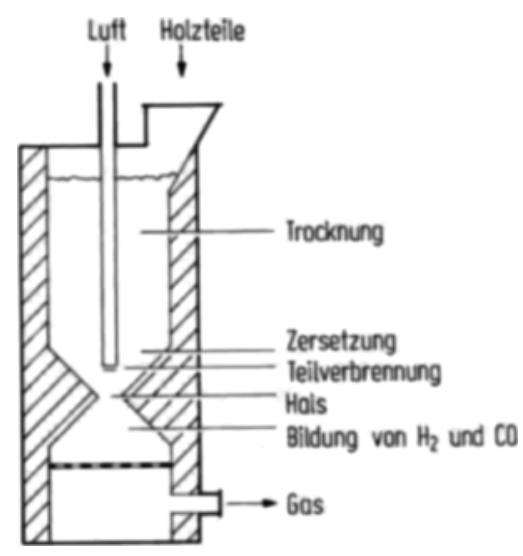

Bild 1. Schema des Gleichstrom-Festbett-Vergasers Fig. I. Co-current moving bed gasifier 
aktionen mit Sauerstolf einen starken Anstieg der Temperatur bis ca. $1700 \mathrm{~K}$ zur Folge. Abgesehen von der Wärmeerzeugung ist die Umwandlung aller kondensierbaren, organischen Produkte (Teer) aus der Verschwelungszone eine wichtige Funktion der Oxidationszone. In einem richtig entworfenen Vergaser sind Holzkohle und ein heißes Gas, das reichhaltig an $\mathrm{CO}_{2}$ und $\mathrm{H}_{2} \mathrm{O}$ ist, jedoch keinen Teer enthält, die Produkte. In der Reduktionszone wird die fühlbare Wärme der heißen Holzkoble und Gase in der endothermen Reaktion von $\mathrm{CO}_{2}$ und $\mathrm{H}_{2} \mathrm{O}$ mit Kohlenstoff absorbiert. Folglich wird ein Gas hergestellt, das $\mathrm{N}_{2}, \mathrm{H}_{2}, \mathrm{CO}_{2}, \mathrm{CO}, \mathrm{H}_{2} \mathrm{O}$ und etwas $\mathrm{CH}_{4}$ enthält.

Die Beschreibung von klassischen Vergasern kann aus der Literatur entnommen werden.

Im folgenden werden die Grundbegriffe der Verfahrenstechnik kurz zusammengefaßt und durch die neuesten Ergebnisse, in bezug auf die Verschwelungszone, erweitcrt. Weiter werden Aspekte des Maschinenbaus diskutiert und schließlich ein Vergaser für verschiedene Zwecke und Kapazitäten beschrieben.

\section{Grundbegriffe der Verfahrenstechnik}

Bei vorausgesetztem chemischem Gleichgewicht und Wärmeverlust können thermodynamische Modelle benutzt werden, um aus den Brennstoffeigenschaften die Zusammensetzung der Produktgase zu berechnen. Für den Entwurf des Vergasers sind komplexere Modelle, welche die Geschwindigkeit der verschiedenen chemischen und physikalischen Prozesse einbeziehen, erforderlich.

\subsection{Verschuelung}

Nachdem ein Brennstoffteilchen getrocknet ist, beginnt es zu verschwelen. Organische Gase und Flüssigkeiten werden herausdestilliert und der feste Rückstand erreicht einen höheren Kohlenstoffgehalt. Um ein teerfreies Produktgas zu erhalten, muß der Verschwelungsproze $\beta$ in der Oxidationszone, im sog. Halsteil, abgeschlossen scin. Gelangen unvollständig verschwelte Teilchen in die Reduktionszone, entsteht Teer, der zum größten Teil nicht umgewandelt im Produktgas verbleibt. Teer, der in oder über dem Halsteil produziert wurde, wird gekrackt, und/oder verbrannt, wenn der Lufteinlass und der Hals richtig entworfen sind.

Der Verschwelungsprozeß großer Teilchen wird, sowohl durch die Kinctik der Verschwelung als auch durch das Eindringen von Wärme in das Teilchen gesteuert. Kinetische Messungen für Holz wurden umfassend veröffentlichı. Für Holz, Papier und DRDF ( = Densified Refuse Derived Fuel $)^{1}$ wurden thermogravimetrische Analysen durchgeführt. Die Resultate, basierend auf einer einfachen Reaktions-Geschwindigkeitskonstanten 1. Ordnung:

$\frac{\delta m}{\delta t}=k\left(m-m_{\mathrm{c}}\right) \exp \left(-E_{l} R T\right)$

sind in Bild 2 dargestellt.

\footnotetext{
1 Pelletierter, aus Müll gewomnener Brennstoff

a Fourier-Zahl

$d_{\mathrm{p}}$ Teilchen-Durchmesser, $\mathrm{m} \quad R \quad$ Gasgehalt, $\mathrm{J} / \mathrm{mol} \mathrm{K}$

$E$ Aktivierungsenergie. $\mathrm{J} / \mathrm{mol}$

$t$ Zeit,

Reaktionsgeschwindigkeitsk onstante

$m$ Masse, $\mathrm{kg}$

$m_{\mathrm{b}}$ Anfangsmasse, $\mathrm{kg}$

$T$ Temperatur, $\mathrm{K}$

$x, y, z$ Raumkoordinaten, $\mathrm{m}$

$X$ Umwandlung

$Z$ Entfernung vom Lufteinla $B, m$
}

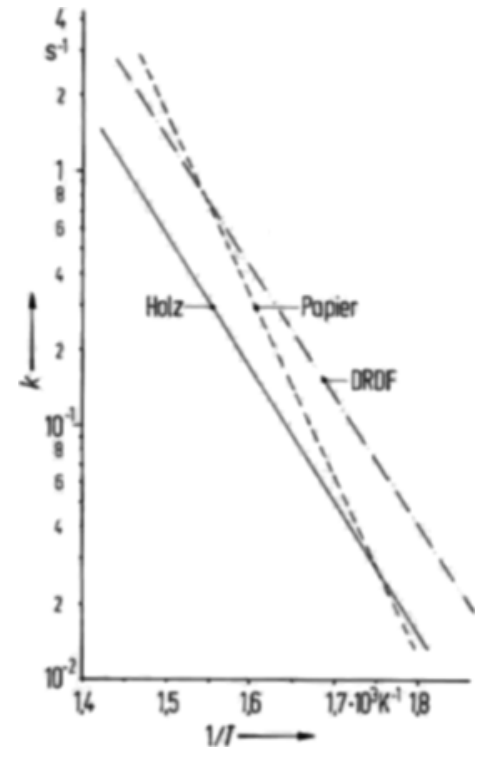

Bild 2. Arrhenius-Diagramm nach thermogravimetrischer Analyse Fig. 2. Arrhenius-plots from TGA measurement

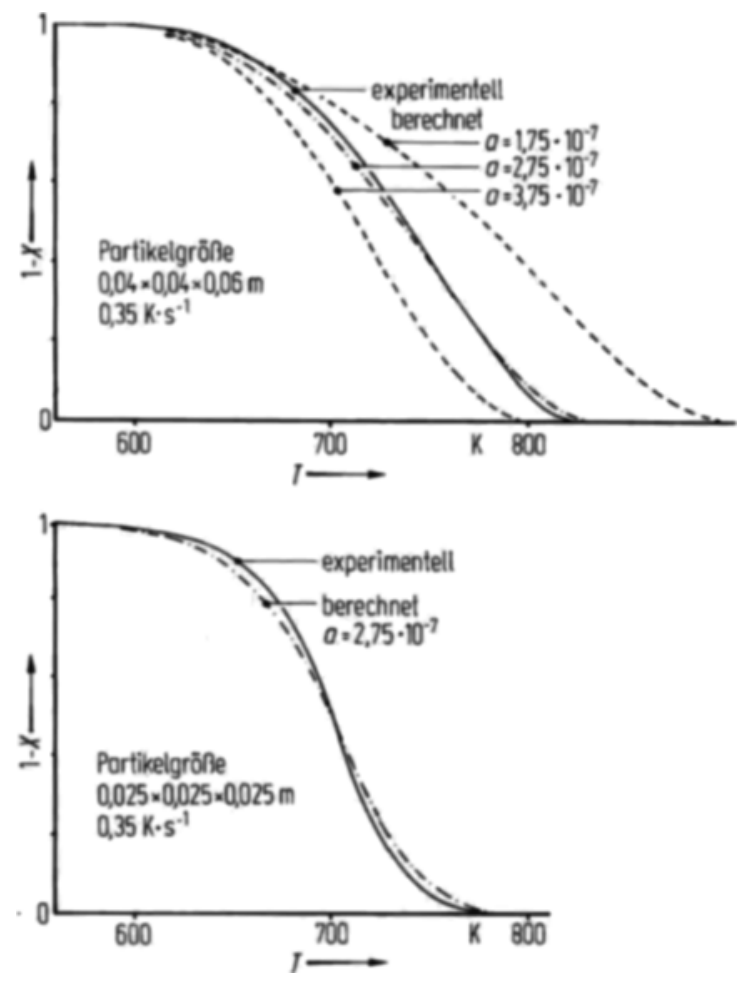

Bild 3. Berechnete und experimentell ermittelte Verläufe bei der pyrolytischen Umwandlung von Holzteilchen in einer Thermowaage Fig. 3. Calculated and experimental pyrolysis conversion for wood particles heated up in a thermobalance

Obgleich die Wärmeeindringung ziemlich kompliziert ist, u.a. aufgrund sich verändernder physikalischer Eigenschaften während der Umwandlung, wird hier eine einfache, dreidimensionale Fourier-Wïrme-Eindringungs-Funktion angenommen

$$
\frac{\delta T}{\delta t}=a\left(\frac{\delta^{2} T}{\delta x^{2}}+\frac{\delta^{2} T}{\delta y^{2}}+\frac{\delta^{2} T}{\delta z^{2}}\right)
$$

Die thermische Diffusivität wird somit hinsichtlich Umwandlung, Richtung und Erwärmungsgeschwindigkeit als konstant angenommen.

In Bild 3 werden Ergebnisse für Holzschnitzel in einer Thermowaage gezeigt. Die Temperatur der Außenseite des 
Teilchens steigt linear mit der Zeit an. Die Gewichtsabnahme wurde sowohl experimentell gemessen als auch mittels einer Integration über die örtliche Umwandlung innerhalb des Teilchens errechnet. Die thermische Diffusivität in der Fourier-Gleichung wird durch die Mcthode der klcinsten Quadrate bestimmt. Dieser numerische Wert trifft für verschiedene Erwärmungsgeschwindigkeiten und Teilchengrößen zu und hängt nur vom Brennstofftyp ab.

Der Temperaturanstieg für ein Teilchen innerhalb des Vergasers verläuft jedoch nicht, wie in thermogravimetrischen Experimenten. linear. Aus experimentellen Temperaturmessungen über der Oxidationszone und der Teilchengeschwindigkeit, kann das Temperaturprofil der Außenseite des Teilchens wie folgt beschrieben werden:

$$
T=T_{0}+\alpha \exp (\beta t)
$$

Die Werte für $\alpha$ und $\beta$ sind mit der Methode der kleinsten Quadrate mit experimentell gemessenen Temperaturen und Feststofrströmungen berechnet. Die örtliche Umwandlung innerhalb eines Teilchens kann nur anhand einer analytischen Lösung durch Fourier-Analyse des Integrals über Wärmeeindringung und Kinetik berechnet werden:

$\ln =\left(\frac{m-m_{\mathrm{e}}}{m_{\mathrm{b}}-m_{\mathrm{e}}}\right)=k \int_{0}^{1} \exp (-E / R T) d t$

mit $T$ als $f(x, y, z, t)$.

Es wird angenommen, da $B$ die maximale Kapazität des Vergasers erreicht ist, wenn die Verschwelungs-Umwandlung des Brennstoffteilchens zu $99 \%$ auf der Höhe des Lufteinlasses stattgefunden hat. Für den Fall der Holzvergasung in einem klassischen Imbert-Vergaser wurde die VerschwelungsUmwandlung eines Teilchens ( $45 \mathrm{~mm} \times 60 \mathrm{~mm} \times 80 \mathrm{~mm}$ ) bei maximaler Kapazität berechnet (Bild 4).

Da es unmöglich ist, den Vergaser rasch genug abzukühlen, kann dieses Profil nicht experimentell bestätigt werden. Hier können nur Experimente, bei denen die Teerproduktion mit der Erhöhung der Kapazität stark ansteigt, herangezogen werden, um die beschriebenen Phänomene zu bestätigen.

Schläpfer beschreibt gut dokumentierte Versuche mit einem klassischen Imbert-Vergaser. Er berichtet, daß die Kapazität. infolge der Teerproduktion, für Holzstücke von $45 \mathrm{~mm} \times 60 \mathrm{~mm} \times 80 \mathrm{~mm}$ auf $50 \mathrm{~kg} / \mathrm{h}$ begrenzt ist. Unsere Modellberechnungen sagen ein maximales Fassungsver. mögen von $47,9 \mathrm{~kg} / \mathrm{h}$ voraus. In einer Versuchsanlage der Twenter Universität für Technologie wurde die Kapazität von $18 \mathrm{~kg} / \mathrm{h}$ auf $23 \mathrm{~kg} / \mathrm{h}$ mil Holzstücken von $20 \mathrm{~mm} \times 20 \mathrm{~mm} \times 20 \mathrm{~mm}$ erhöht. Dies führte zu einem Anstieg der Teerproduktion mit dem Faktor 5. Die berechnete maximale Kapazität betrug $19,8 \mathrm{~kg} / \mathrm{h}$. Ähnliche Erscheinungen wurden für DRDF ( $20 \mathrm{~mm}$, rd. $40 \mathrm{~mm}$ lang) beobachtet, wenn man die Kapazität in einer E.E.E.-Versuchsanlage mit ständiger Aschenbeseitigung von 30 auf $42 \mathrm{~kg} / \mathrm{h}$ erhöhte. Die errechnete maximale Kapazität betrug hier $32,5 \mathrm{~kg} / \mathrm{h}$. Demnach beschreibt das hier vorgestellte Modell das maximale Fassungsvermögen eines Vergasers erstaunlich gut.

\subsection{Oxidation}

Der Oxidationsprozeß ist die Wärmequelle für den Verschwelungsprozeß über, und den Reduktionsprozeß unter dem Lufteinlaß. Neben der Wärmeerzeugung ist es die Hauptaufgabe dieser Zone, die kondensierbaren, organischen Verschwelungsprodukte ( $z$. B. Teer) in nicht-kondensierbare Stoffe umzuwandeln. Letztere Funktion wird haupt-

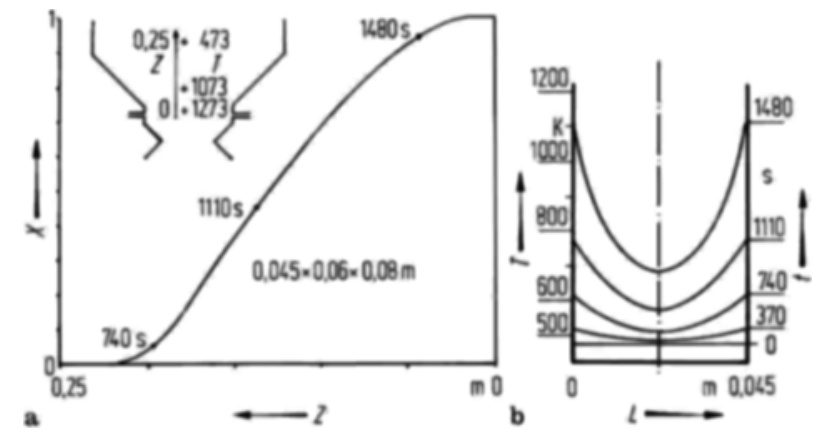

Bild 4. a Pyrolysediagramm für einen Imbert-Vergaser. Die pyrolytische Umwandlung wurde auf der Basis experimentell ermittelter Temperaturen bei maximaler Kapazität berechnet, b Temperaturprofil in einem Holzteilchen, das sich durch die Mitle eines ImbertVergasers bewegt

Fig. 4. a Pyrolysis in an Imbert gasifier. Pyrolysis conversion is calculated based on experimental temperatures at maximum capacity, b temperature profile in a wood particle flowing through the centre of an Imbert wood gasifier

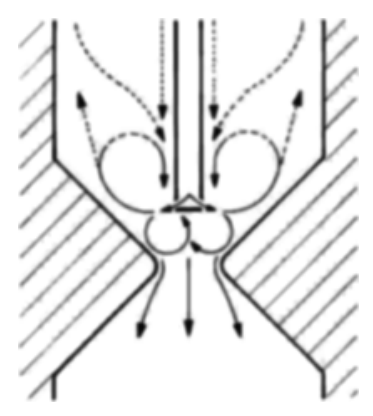

Bild 5. Schema der Gasbewegungen im Brennkammerhals des Vergasers

Fig.5. Gasflow patterns in the throat section of a gasifier

sächlich bestimmt durch die Gas-Strömungs-Muster und das Temperaturprofil innerhalb des Vergasers. Aufgrund von Tracer- $\left(\mathrm{CH}_{4}\right)$-Injektionsversuchen und Kaltströmungs-Modell-Versuchen werden die Strömungs-Muster in Bild 5 vorausgesetzt. Die Größe der Strömungsmodelle hängt weitgehend von Faktoren wie Teilchengröße und -verteilung, Lufteinlaßgeschwindigkeit etc. ab. Daher ist der genaue Entwurf des Halses für jeden Brenrstoff mehr oder weniger empirisch. Das kreisrunde Brennkammer-Hals-Konzept ermöglicht die Erhöhung des Volumens und des Querschnittes des Halses, während die Größe des Strömungs-Musters unverändert bleibi.

Auf diese Weise können Sägespäne, Holzspäne und landwirtschaftliche Abfallprodukte teerfrei vergast werden und es ist möglich, den Vergaser bis zu einer Kapazität von wenigstens $1000 \mathrm{~kg} / \mathrm{h} \mathrm{zu}$ vergrößern. Experimentelle Ergebnisse sind in Tabelle 1 aufgeführt.

\subsection{Redukion}

Durch die Reduktion von $\mathrm{CO}_{2}$ und $\mathrm{H}_{2} \mathrm{O}$ mit Kohle erhält man die brennbaren Komponenten $\mathrm{CO}$ und $\mathrm{H}_{2}$ im Produktgas. Dabei wird die fühlbare Wärme aus der Oxidation verbraucht.

Die Geschwindigkeit des Verfahrens kann begrenzt werden durch:

die Massen- und Wärmeübertragung von der Gasphase zu den Teilchen,

die Diffusion der gasförmigen Reaktionsteilnehmer in das poröse Teilchen, 
Tabelle 1. Masse und Energiegebalt verschiedener Brennstoffe

\begin{tabular}{|c|c|c|c|c|c|}
\hline & \multicolumn{5}{|c|}{ Festbrennstoffe } \\
\hline & $\begin{array}{l}\text { Holz-Hack- } \\
\text { schnitzel }\end{array}$ & $\begin{array}{l}\text { Siggespiine } \\
\text { Hobelspäne }\end{array}$ & DRDF & $\begin{array}{l}\text { Hafer- } \\
\text { spreu }\end{array}$ & $\begin{array}{l}\text { Mais- } \\
\text { kolben }\end{array}$ \\
\hline Mittlere Korngröße, $\mathrm{cm}$ & 1,0 & 0,3 & 3,0 & 0,2 & 5,0 \\
\hline Feuchte, Gew.-\% & 18,0 & 8,0 & 10,0 & 15,0 & 8,0 \\
\hline Aschengehalt, Gew:- $\%$ & 0,2 & 0,2 & 26,5 & 5,0 & 3,8 \\
\hline \multicolumn{6}{|l|}{ Einsatz, kg/h: } \\
\hline Feststof [ & 32,0 & 18,4 & 32,0 & 26,7 & 24,9 \\
\hline Luft & 57,8 & 28,11 & 40,78 & 45,9 & 49,8 \\
\hline \multicolumn{6}{|l|}{ Ausbeute, $\mathrm{kg} / \mathrm{h}$ : } \\
\hline Trockengas & 81,53 & 44,44 & 55,92 & 60,3 & 68,3 \\
\hline Kondensat & 7,65 & 1,57 & 6,89 & 10.7 & 5,2 \\
\hline Asche & - & 0,5 & 9,97 & 1.5 & 1.1 \\
\hline \multicolumn{6}{|c|}{ Reingas-Zusammensetzung, Vol.- $\%$ : } \\
\hline Co & 17,1 & 17.6 & 15,9 & 24,3 & 16,5 \\
\hline $\mathrm{H}_{2}$ & 18.6 & 18,2 & 12,0 & 11.1 & 16.9 \\
\hline $\mathrm{CO}_{2}$ & 14,4 & 16,4 & 14,8 & 8,4 & 14,1 \\
\hline $\mathrm{N}_{2}$ & 47,6 & 44,2 & 53,8 & 54.9 & 51,3 \\
\hline $\mathrm{CH}_{4}$ & 2,3 & 3.1 & 2,4 & 2,2 & 2,2 \\
\hline $\mathrm{C}_{2}+$ & - & 0,5 & 1,1 & 0,2 & 0.1 \\
\hline \multicolumn{6}{|l|}{ Energiegehalt: } \\
\hline Brennstoffe, MJ/kg & 14,69 & 16,79 & 13,28 & 16.0 & 16,85 \\
\hline Reingas, $\mathrm{MJ} / \mathrm{kg}$ & 4,33 & 5,02 & 4,02 & 4,77 & 3,99 \\
\hline Therm. Effiz., \% & 75,1 & 72,0 & 53,0 & 67,3 & 65,0 \\
\hline
\end{tabular}
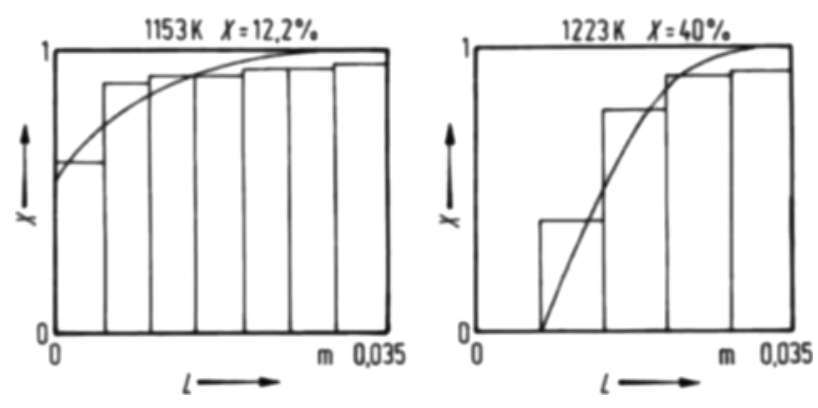

Bild 6. Lokale Kohleumwandiung in einem Holzkohlenpartikel, vergast mit $\mathrm{CO}_{2}$ beginnend bei $L=0$. Das Stufendiagramm zeigt die experimentell ermittelten, die Kurve die berechneten Werte

Fig. 6. Local carbon conversion in a linear wood char particle gasified with $\mathrm{CO}_{2}$ from $L=0$. The stepped curve is experimental, the other calculated
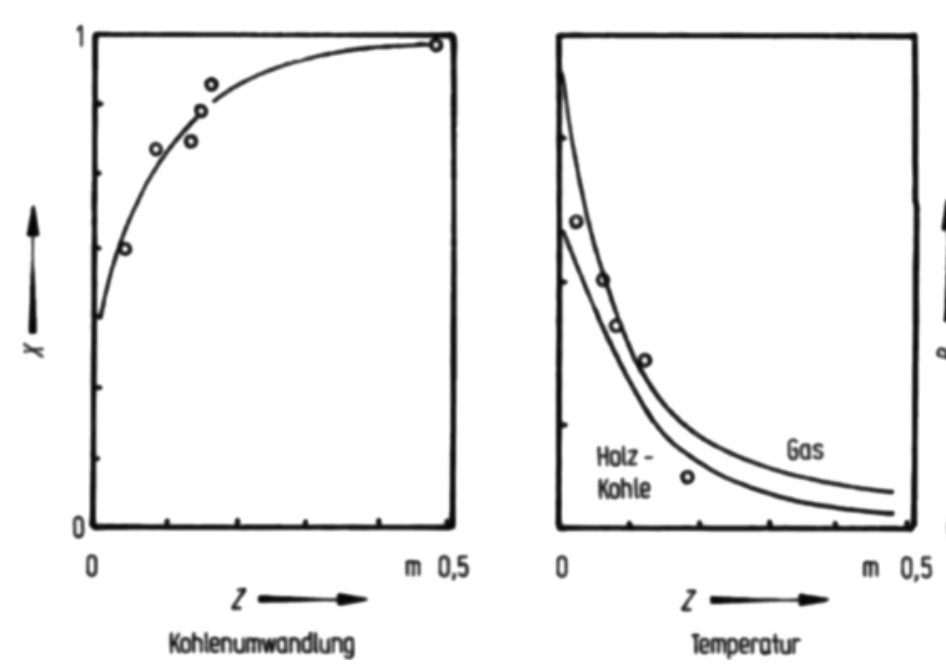

die chemische Reaktion an der aktiven Oberfläche des Teilchens,

die Diffusion und Konvektion der gasförmigen Produkte aus dem Teilchen in die Gasphase.

Aur einer Thermowaage wurde die Kinetik der Reaktionen bestimmt. Für die letzten drei die Geschwindigkeit begrenzenden Schritte wurde ein Teilchen-Modell entwickelt und verifiziert. Das Modell berücksichtigt, daß sowohl der Diffusionskoeffizient als auch die chemische Reaktionsgeschwindigkeit eine Funktion der örtlichen Kohlenstoffumwandlung und damit auch eine Funktion von Zeit und Ort innerhalb des Teilchens ist. Das Modell beschreibt auch die Schwindung der Teilchen während der Umwandlung. Es wurden Teilchen-Experimente durchgeführt, um die Richtigkeit des Modells zu beweisen. Typische Beispiele sind in Bild 6 dargestellt.

Bild 7. Berechnete und experimentell ermittelte Verläufe in der Reduktionszone eines Vergasers

Fig. 7. Calculated and experimental profiles in the reduction zone of a gasifier 
Das Tcilchen-Modell ist kombiniert mit Wärme- und Massen-Übertragungsglcichungen, Wärme- und Massengleichgewichten und Strömungsgleichungen für die Gas- und Festphase, wobei letztere bestimmt wird durch das Schwinden der Teilchen und die Aschenbeseitigung. Experimentelle und berechnete Daten sind in Bild 7 angegeben. Demnach kann das Reduktionszonen-Modell benutzl werden, um dic Länge und das Volumen dieser Zone zu berechnen. Auch sagt es dic Temperaturen im Vergaser sehr gut voraus. Dies ist wichtig für Brennstofle, die Asche mit einer Tendenz zur Zunder-oder Schlackenbildung haben. Für die verschiedenen Schritle des Verfahrens wurden Modelle entwickelt, die, basierend auf den Brennstoffeigenschaften, die Prozeß-Daten und -Begrenzungen beschreiben. Diese Brennstoffeigenschalten können aus thermogravimetrischen Analysen entnommen werden. Damit kann ein Verfahren für die verschicdenen Brennstoffe entwickelt werden, dessen Realisierung jedoch noch sehr vicle Aspekte des Maschinenbaus beinhaltet.

\section{Maschinenbauliche Aspekte}

Die Grundabmessungen des Vergasers wurden aus den Bcrechnungen der Verfahrenstechnik entnommen. Um den Vergaser konstruicren zu können, müssen die Regeln des Malschinenbaus zugrundegelegt werden, mit Rücksicht auf Brennstofl-Handhabung, Vergaser-Konstruktion. Handhabung der Asche, die Behandlung und schließlich die Nutzung des Gases.

\subsection{Handhabung des Bremustoffs:}

Aus dem Brennstofflager muß der Brennstoff entweder automatisch oder manuell in den Vergaser gebracht werden. Da sowohl ein Eindringen von Luft in den Vergaser, als auch ein Austreten von Gas aus dem Vergaser aus Sicherheitsgründen nicht akzeptabel ist, muß die Zuleitung des Brennstoffs so gasdicht wie möglich erfolgen. Bei kleinen Anlagen kann cine gasdichte Tür benutzt werden, solange das einzuleitende Brennstoffvolumen relatjv klein ist und die Tür daher nur für kurze Zeit geöffnet werden muß3. Dies trif̂̀t generell für manucll betriebene Vergalser zu. Für klcine, automatisch betriebene Anlagen können doppeltürige Spüikanäle oder ein, mit einer Tür kombinierter Schneckenförderer eingesetzt werden.

Die gasdichten Türen werden zwar durch Teer und klebende Brennstoffteilchen angegriffen, sind aber billig und einfach zu konstruieren. Größere Anlagen benötigen allerdings einen so großen Volumenstrom des Brennstoffes, besonders im Fall von Sägespänen und landwirtschaftlichem Abfall, daß rotierende Spülkanäle unvermeidlich sind. Diese Spülkanäle müssen bei verschiedenen Temperaturen gasdicht sein. gegen Verschmutzung mit Teer und Staub immun scin, bei feinen und bei groben Materialien, also auch für zähe und faserige Biomasse und deren Verunreinigungen anwendbar sein

Ähnliche Probleme treten bei der Brennstofflagerung und am Bunker des Vergasers auf, wenn AbCälle extrem schlechte Strömungseigenschaften zeigen. Für die Brennstoftlagerung gibı es in der Literatur viele Lösungsvorschläge. Für den Vergaserbunker jedoch herrschen andere Bedingungen. In den meisten Fällen reichen Rührwerke und Brückenbrecher aus, um die Strömung der Feststoffe zu verbessern, aber sie erfordern sich bewegende Apparaturen innerhalb des Vergasers.

Sich drehende Apparaturen müssen natürlich gasdicht eingeführt werden und auch unter hoch-korrosiven Bedingungen einsatzlähig bleiben.

\subsection{Materialauswahl für den Vergaser}

Das größte Problem hierbei ist, passendes Material für den Hals und wenn nötig, hitzebeständige Rührwerke auszuwählen.

Um im Hals eine hohe Temperatur zu erreichen, ist es nötig. diesen zu isolieren. Auch wenn der klassische Imbert-Vergaser mit cinem Hals aus Stahl arbeitet, ist es schwierig, damit ausreichende Lebensdauer, hobe Leistungsfähigkeit und einen teerfrcien Betrieb zu erzielen. Deshalb wird ein isolicrter Keramik-Hals bevorzugt. Die Keramik muß sowohl den Temperaturen in der Oxidationszone als auch dem thermischen Schock. der während des Startens auftritt. standhalten Die Düsen des Lufteinlasses sind mehr oder weniger luftgekühlt und können aus passendem Stahl oder Keramik hergestellt werden. Alle Materialien, die innerhalb des Vergasers eingesetzt werden, müssen resistent gegen Säure-Komponenten wie Teer. Essigsäure ctc. scin.

\subsection{Beseitigung der Asche}

Die Asche muß periodisch oder kontinuierlich beseitigt werden. Dies hängt von der Kapazität des Vergasers und dom Aschengehalt des Brennstoffes ab. Das AschenbeseitigungsSystem entfernt nicht nur die Asche, sondern muß auch dazu imstande sein, den Druckabfall über dem Vergaser zu reduzieren, den Staubgchalt in Brennstoffgas auf ein Mindestmaß zu beschränken, sowic entweder die Schlacke- und Zunderbildung zu verhindern, oder diese Produkte zu zerkleinern und zu verhindern, daß Löcher in das Brennstoffbett unterhalb des Halses gebrannt werden.

Für die Holz-Vergasung in kleinem Maßstab sind sehr einfache Lösungen, wie z. B. ein Schüttelrost mit gasdichtem Aschenbehälter gefunden worden. Für Brennstoffe mit höherem Aschengehalt wurden häufig exzentrisch rotierende Roste vorgeschlagen, die dem Kerpely-Rost der Kohlen-Vergaser weitgehend gleichen. Mit einem solchen Rost hat Jenkins Brennstoffe mit einem maximalen Aschengehalt von ungefäh $6 \%$ vergast. Dic E.E.E.-Vergaser verwenden, abhängig vom Brennsioffyp. modifizierte, exzentrisch rotierende Vorrichtungen. Dadurch ist es möglich, DRDF mit einem hohen Silika-Aschengehalt von $26 \%$ auf a kzeptable Weise zu vergasen (Tábelle 1). Bei den meisten Anlagen bevorzugt man, die Asche aus dem Vergaser zu spülen. Bei geringer Kapazität reichen ein Aschenbehälter und ein gasdichtes Ventil aus, be größerer Kapazität wird ein rotierender Spülkanal eingesetzt.

\subsection{Gasbehandlung und Gasnutzung}

Die Gasbehandlung mul3 so entworfen werden, daß sie sowohl den Anforderungen der Gasnutzer (z. B. Boiler, Motor), als auch der Umwelt gerecht wird. Viele Probleme bei der Gasbehandlung haben ihre Ursache in einem fehlerhaften Vergaser-Entwurf. Besonders die Beseitigung von Teer und Staub kann durch einen geeigneten Vergaser-Entwurl stark vereinfacht werden. Wie von Hos bewiesen wurde, können auch saure Gase, wie Chlorwasserstoff während der Vergasung beseitigt werden. Dies geschieht durch Zuführung sehr kleiner Mengen z. B. von Kalkstein in den Vergaser.

Für direkte Erwärmungsprozesse ist eine Gasbehandlung unnötig, sofern ein Schwachgas-Brenner benutzt wird. Diese Brenner können so entworfen werden, daß sie heißes Gas, das kleine Mengen Tecr und Staub enthäłt, annehmen. Hierbej ist der Umbau von konventionellen oder bestehenden Boilern 
und Feuerungen möglich. Für die Kraft oder die Wärme/ Kraft-Erzeugung sind Gaskühlung und reinigung unumgänglich. In diesem Fall wird das Gas in einem Gas- oder Dieselmotor verbrannt, wodurch Kraft und Wärme entstehen. Der Motor benötigt kaltes Gas, um den Füllungsgrad des Zylinders und sauberes Gas, um die Lebensdauer des Motors zu erhöhen. Die Kühlung kann auf 2 Arten stattfinden: durch einen mit Wasser oder Luft gekühlten Wärmeaustauscher, oder durch die Einspritzung von kaltem Wasser in das Gas. Für die Gasreinigung kann eine Kombination aus Zyklon, Naßabscheider, Naßfilter, Trockenfilter und/oder Elektrofilter benutzt werden. Produziert der Vergaser etwas Teer, so sind diese Systeme für Verstopfungen anfällig. Vorausgesetzt, da 3 ein passender Gas/Luft-Mischer eingesetzt wird, können im Prinzip alle Gas- und Dieselmotoren mit sauberem, kaltem Holzgas angetrieben werden. Dieselmotoren können entweder als Zweistoffmotoren, die $50 \ldots 75 \%$ Dieselöl ersetzen, oder als Pilotinjection-Motoren betricben werden, die dann $80 \ldots 90 \%$ Dieselöl ersetzen. Der Umbau zu einer Pilotinjection ist in jedem Fall komplizierter und teurer, als der Umbau zum Zweistoffmotor. Der direkte Gebrauch von Gasturbinen müßte prinzipiell möglich sein, wurde aber in der Literatur bis jetzt noch nicht beschrieben.

\subsection{Sicherheit}

Eine Vergasungsanlage produziert giftiges Gas (Kohlenmonoxid). Mit Luft vermischt, ist dieses Gas explosiv. Der untere Explosions-Grenzwert liegt bei schätzungsweise $4 \mathrm{Vol}-\%$ Sauerstoff im Gemisch. Daher muß3 das System völlig gasdicht sein, so daB weder Gas entweichen, noch Luft angesaugt werden kann. Während des Startens wird das System mit Luft aufgefüllt und, soweit dann nicht mit inaktivem Gas gespült wird, besteht die Möglichkeit einer Explosion. Um diese Gefahr so klein wie möglich zu halten, ist es eine der wichtigsten Aufgaben, das freie Gasvolumen des Systems auf ein Minimum zu beschränken.
Bei dem direkt mit Wasser gekühlten System gibt es keine Explosionsgefahr. Solite es jedoch nicht möglich sein, dieses System im Vergaser und bei der Aschenbeseitigung zu verwenden, muß der Gasbehälter so konstruiert sein, daß er dem maximalen Explosionsdruck von 6 bar standhält. Auch Sicherheitsventile oder Explosionsluken können verwendet werden, wobei dann allerdings die Gefahr von Gas-Emissionen besteht.

Besondere Vorsicht muB man bei handbefüllten Vergasern, die nur eine gasdichte Tür haben, walten lassen. Steht die Tür offen, können Flammen entweichen und nachdem die Tür dann geschlossen wurde, ist es möglich, daß es zu einer kleinen Bunker-Explosion kommt. Daher muß diese Tür nach dem Prinzip der Sicherheitsventile konstruiert sein. Allerdings ist eine Leckage in der Abdichtung auch dann noch möglich, wenn Staub und Brennstoffteilchen an ihr kleben bleiben. Diese Probleme sind dadurch zu lösen, daß die Bunker-Auffüllung mit ausreichender Betthöhe über dem Hals stattfindet. Nach kurzer Trainingsperiode kann eine manuelle Anlage sicher betrieben werden.

\section{E.E.E.-Vergasungsanlagen}

Die Entwicklungsarbeit wurde mit zwei Versuchsanlagen durchgeführt. Eine Anlage wird handbefüllt betrieben und hat eine Kapazität von $50 \mathrm{~kg} / \mathrm{h}$ Abfall. Die andere arbeitet vollständig automatisch mit einem Fassungsvermögen von $300 \mathrm{~kg} / \mathrm{h}$. Besondere Aufmerksamkeit mußte gerichtet werden auf die Entwicklung des kreisrunden Brennkammer-Halses für möglichst viele, verschiedene Brennstoffarten und zur VergröBerung geeignet, aul ein ständig rotierendes Aschenbeseitigungs-System und auf den vollständig automatischen Betrieb: Automatische Start- und Schließverfahren, gasdichte, rotierende Spülkanäle sowohl für die Brennstoffaufnahme als auch lür die Aschenbeseitigung und Verfahrenskontrolle (u.a. die Einstellung der Kapazität auf den Motorverbrauch).

Tabelle 2. Daten zur Wirtschaftlichkeit einer Vergasungsanlage Bauart E.E.E. Modell 125 unter Zugrundelegung niederländischer Verhältnisse

\begin{tabular}{|c|c|c|c|c|}
\hline & \multicolumn{3}{|c|}{$\begin{array}{l}\text { Reine } \\
\text { Wärmeerzeugung } \\
500 \mathrm{~kW}_{\mathrm{th}}\end{array}$} & $\begin{array}{l}\text { Wärme } / \text { raft- } \\
\mathrm{Koppelung} \\
125 \mathrm{~kW}_{*} / \mathrm{kW}_{\mathrm{th}}\end{array}$ \\
\hline \multicolumn{5}{|l|}{ Kapital- und Aufstellungskosten; US \$ } \\
\hline $\begin{array}{l}\text { Brennstofflagerung } \\
\text { Vergasungsanlage } \\
\text { Umbau des vorhandenen Damplkessels } \\
\text { Wärme/Kraft-Einrichtung } \\
\text { Installationskosten }\end{array}$ & & $\begin{array}{l}10000 \\
40000 \\
20000 \\
- \\
4000\end{array}$ & & $\begin{array}{l}10000 \\
50000 \\
. \\
70000 \\
10000 \\
\end{array}$ \\
\hline \multirow[t]{2}{*}{ Prämie für energiesparende Investition (Holland) $32 \%$} & & $\begin{array}{l}74000 \\
24000\end{array}$ & & $\begin{array}{r}140000 \\
45000 \\
\end{array}$ \\
\hline & & 50000 & & 95000 \\
\hline Nutzung; h/Jahr & 2000 & 6000 & 2000 & 6000 \\
\hline $\begin{array}{l}\text { Abschreibung, } 10 \text { Jabre } \\
\text { Zins, im Mittel } 5 \% \\
\text { Nutzung } \\
\text { Wartung }\end{array}$ & $\begin{array}{l}5000 \\
2500 \\
3000 \\
2000 \\
\end{array}$ & $\begin{array}{l}5000 \\
2500 \\
5000 \\
3000 \\
\end{array}$ & $\begin{array}{l}9500 \\
4750 \\
3000 \\
4000 \\
\end{array}$ & $\begin{array}{l}9500 \\
4750 \\
5000 \\
8000 \\
\end{array}$ \\
\hline Wartung & 12500 & 15500 & 21250 & 27250 \\
\hline \multicolumn{5}{|l|}{ Jährliche Ausbeute } \\
\hline $\begin{array}{l}\text { Wärme } 0,028 \$ / \mathrm{kWh} \\
\text { Strom } 0,08 \$ / \mathrm{kWh} \\
\text { Amortisation Jahre } \\
\text { Kosten je } \mathrm{kWh} \text { ir in US } \$ \\
\text { Kosten je } \mathrm{kWh} \text { in US } \$\end{array}$ & $\begin{array}{l}28000 \\
- \\
3,2 \\
0,0125 \\
-\end{array}$ & $\begin{array}{l}84000 \\
- \\
0,7 \\
0,0005 \\
-\end{array}$ & $\begin{array}{l}11200 \\
20000 \\
9,5 \\
- \\
0,085\end{array}$ & $\begin{array}{l}33600 \\
60000 \\
1,4 \\
- \\
0,036\end{array}$ \\
\hline
\end{tabular}




\section{Wirtschaftliche Aspekte}

Die Investitionskosten für E.E.E.-Vergasungsanlagen hängen in hohem Maß von den Brennstoffeigenschaften und den Brennstoff-Behandlungskosten ab. Da größere Anlagen kompliziertere und teurere Behandlungsgcräte erfordern. bleiben die Investitionskosten pro kW für kleine und große Anlagen mehr oder weniger gleich. Nach einer ersten Berechnung kostet ein vollautomatischer Vergaser für die direkte Erwärmung ca. $75 \$$ pro $\mathrm{kW}_{\mathrm{th}}$. Eine handbefüllte Anlage, inklusive Gaskühlung und Gasreinigung, kostet etwa $400 \$$ je $\mathrm{kW}$ Elektrizität, wobei der Generator-Set mit ca. $350 \$$ je $\mathrm{kW}$ nicht eingerechnet ist.

Eine vollständige Anlage. welche dic Brennstofflagerung. den automatischen Betrieb des Vergasers und eine Kraft/ Wärme-Anlage enthält, wird bei etwa $1000 \$$ je kW liegen.

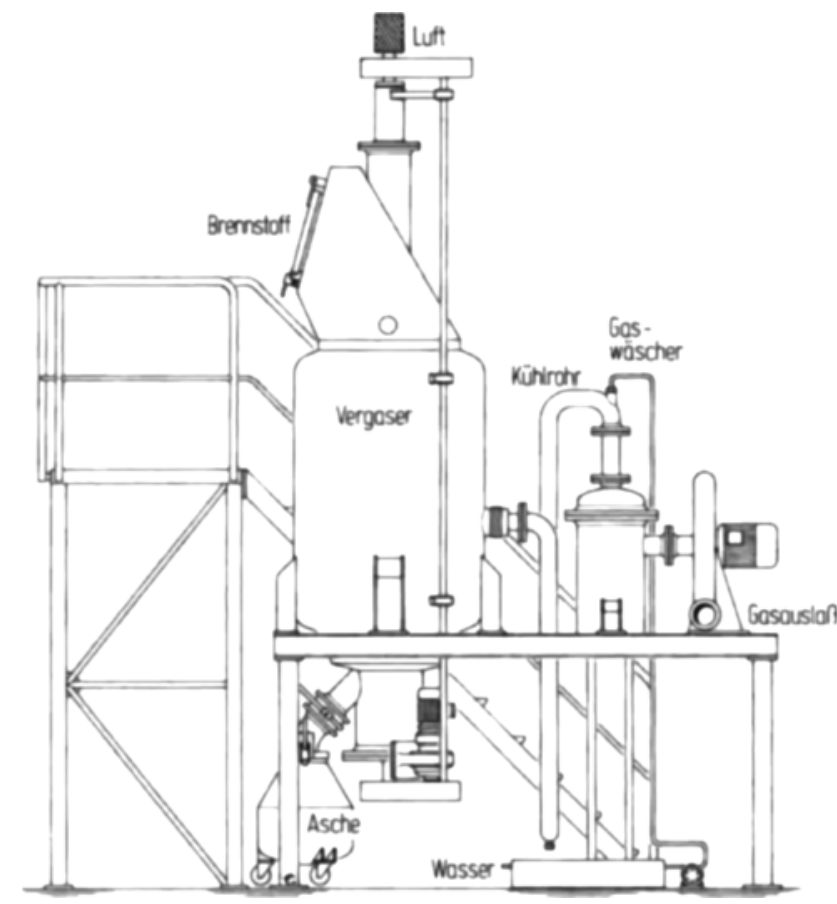

Bild 8. Handbeschick te Vergasungsanlage, Typ EEE 65 , für $65 \mathrm{~kg} / \mathrm{h}$ Abfälle, TeilchengröBe $1 \ldots 50 \mathrm{~mm}$, Aschengehalt bis $30 \%$; automatische Beschickung ist nachrüstbar

Fig. 8. Hand-loaded gasification plant EEE 65 , for $65 \mathrm{~kg} / \mathrm{h}$ of various wastes; particle size 1 .. $50 \mathrm{~mm}$, ash content up to $30 \%$; automatic fuelling is possible
Unter niederländischen, wirtschaftlichen Verhältnissen ist die letztgenannte Anlage für die Holz- und Möbelindustrie vorteilhaft, da sie aus deren Abfällen Energie produzieren kann. Abhängig vom Verwendungszweck, der Anzahl der Betriebsstunden pro Jahr und den Verkaufskosten oder dem Marktwert des Abfalls liegt die Amortisationszeit zwischen I und 5 Jahren (Tabelle 2).

Es hat sich gezeigt, daß die Gleichstrom-Festbett-Vergaser mit einem kreisförmigen Hals und kontinuierlicher Aschenbeseitigung mit verschiedenen Brennstoffarten (Teilchengrößen 1 .. $50 \mathrm{~mm}$, Aschengehalt bis zu 30\%) sehr gut arbeiten. Im Augenblick können Kapazitäten bis $200 \mathrm{~kg} / \mathrm{h}$ gebaut werden, doch werden bereits größere Einheiten entwickelt.

Bis heute wurden drei Vergaser-Grundtypen entwickelt.

1. Eine einfache, handbefüllte Anlage für grobe Brennstoffe (Holzstücke, Maiskolben, etc.) in mehr oder weniger klassischer Technik, bestehend aus einem Vergaser mit zentral befestigtem Lufteinlaß, keramischem Hals, einem Schüttel-Rost, einem Rohkühler, Naßabscheider und Naßfilter, Fassungsvermögen $20 \ldots 100 \mathrm{~kg} / \mathrm{h}$.

2. Ein Vergaser kleinen Maßstabs, für verschiedene Brennstoffe. Diese Anlage ist sowohl handbefüllt, als auch automatisch für ein Fassungsvermögen von $50 \ldots 200 \mathrm{~kg} / \mathrm{h}$ lieferbar. Ein Beispiel zeigt Bild 8 , bestehend aus einer gasdichten Brennstoffaufnahme, Vergaser mit Rüttel-Bunker, kontinuierlicher Aschenbeseitigung in einen Behälter, Rohkühler, Naßabscheider, Naßfilter und Ventilator. Experimentelle Ergebnisse für verschiedene Brennstoffe (Teilchengröße 1 . . $50 \mathrm{~mm}$, Aschengehalt bis zu 30\%) wurden mit der $50 \mathrm{~kg} / \mathrm{h}$ Versuchsanlage erzielt; sie sind in Tabelle I zusammengestellt.

3. Ein automatischer Vergaser für Kapazitäten von $300 \ldots 1000 \mathrm{~kg} / \mathrm{h}$ Holzabfälle. Da die Versuchsanlage ziemliche Material- und Konstruktionsprobleme aufwies und bis Herbst 83 erst 30 Stunden in Betrieb war, können hier keine genaueren Untersuchungsergebnisse angeführt werden. Diese Anlage besteht aus einer automatischen Brennstoff-Aufnahme, einem rotierenden Rührwerk im Bunker, einer kontinuierlichen Aschenbeseitigung mit wassergekühitem Spülkanal, einem Gas-Luft-Wärmeaustauscher, einem Zyklon und einem Wasserring-Kompressor (Bild 9). (1) Die Holzabfälle (Hackschnitzel, Späne, Sägemehl) werden oben in den Vergaser eingeführt. Ein automatisches Ventil sichert die ständige Materialzufuhr, kontrolliert durch einen Niveauanzeiger. (2) Vorgewärmte Luft, die für den Vergasungsprozeß nötig ist, tritt durch ein Rohr in das Reaktorzentrum ein. (3) Ein kreis-

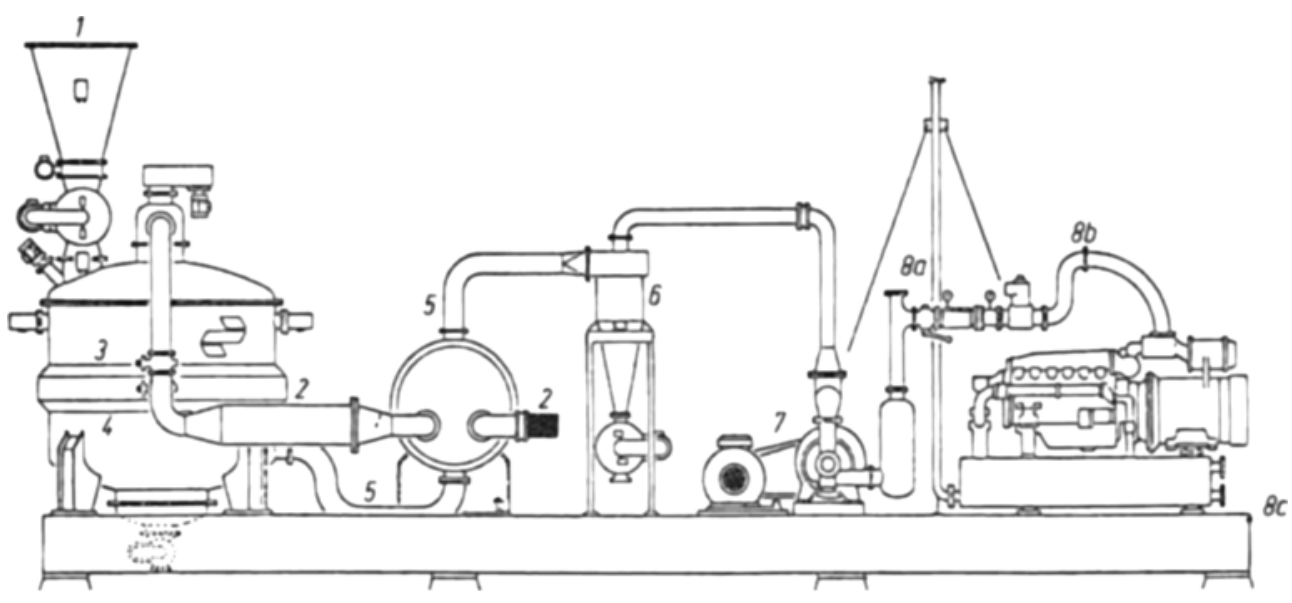

Bild 9. Pilotanlage für die Vergasung von $300 \mathrm{~kg} / \mathrm{h} \mathrm{Holz} \mathrm{in} \mathrm{Betrieb} \mathrm{bei} \mathrm{E.E.E.} \mathrm{in} \mathrm{Oldenzaal,} \mathrm{Holland}$ Fig. 9. Pilot plant for the gasification of $300 \mathrm{~kg} / \mathrm{h}$ wood at Oldenzaal, Netherlands 
rundes Feuerbett wird dicht über dem Brennkammerhals aufgebaut. Hier wird das Holz in Kohle, Teer, Kohlendioxid und Wasscrdampf ungewandelt. Durch die hohen Temperaturen wird der Teer gekrackt und verbrannt. Einer der Vorteile des E.E.E.-Vergasers besteht in der kompletten Unwandlung des Teers. (4) Durch die Reaktion von Kohle mit Kohlendioxid und Wasserdampf, wird brennbares Gas, das Wasserstoff und Kohlenmonoxid enthält. erzeugt. (5) Das Generatorgas wird mit einer Temperatur von 500 C zurückgeführt und in einem Wärmeaustauscher abgekühlt. Die Wärme wird zurückgewonnen für die Vorwärmung der Luft, die für den Vergasungsprozeß erforderlich ist (siehe 2). Das Generatorgas wird au $200 \mathrm{C}$ abgekühlt (6) Das Gas wird auf $30^{\circ} \mathrm{C}$ gekühlt und nach der Staubbeseitigung gewaschen. (7) Das saubere. kalte Generatorgas wird zu einer Kesselanlage oder zu cinem Motor transportiert. (8a) Wird das Generatorgas in ciner Kesselaniage verbrannt, so entstehen Wasserdampf oder heißes Wasser. (8b) Wird das Gas in einem Motor verbrannt, so wird dadurch Kraft erzeugt. (8c) Die Wärme des Kühlwassers und der Abgase kann für anderweitige Erwärmungsprozesse verwendet werden.

Nach weiterer Entwicklung wird diese Anlage in der Lage sein, auch andere Brennstoffe zu vergasen.

Hier wird nur die Art der Gaserzeugung beschricben. Natürlich können diese Anlagen auch für direkte Erwärmungsprozesse verwendet werden. In diesem Fall sind Gaskühlung und Gasrcinigung nicht erlorderlich und anstatt eines Sauggebläses wird ein Luftkompressor am Lufteinlaß eingesetzt.
Schweingruber, F. H.: Der Jahrring. Standort, Methodik, Zeit und Klima in der Dendrochronologie. 234 S. 283 Bilder, 317 Zeichnungen, Pappband. Bern, Siutgart: Paul Haupt Verlag. 125.- DM

Das Buch bietel eine umlassende, tielgehende Darstellung über den Jahrring als Grundlage der Dendrochronologie. Dabei wird auch dem methodischen Vorgehen dieses Wissenschaliszweiges groBer Raum geschenkt. Das Buch ist in folgende Kapitel gegliedert: Herkunfi des Materials, Analyse des Materials, lahrringwachstum und Standort. Angewande Dendrochronologie, Geschichte der Dendrochronologic. 7ahlreiche Fotos sowie gut gelungene zeichnerische Darstellungen und Übersichlen ergänzen den of sehr knapp gehaltenen Text. Dennoch oder gerade dadurch stellt das Buch an die Komzentration des Interessierten hohe Ansprüche. Beim Sudium dieses Buches spürt man immer wieder das grobe Engagenent des Autors. Besonders deutlich wird dies im Zusammenhang mil der Radiodensitometric aber auch bei der Entwicklung von Theoricn (wic 2. B. auf S. 142 145). Die Vielfall der Auskinnte. Welche die Analyse von Jahrringen vermitteln kann, wird in der angewandten Dendrochronologie deutlich. Wenn Zusammenhänge zur Siedlungs- und Kunstgeschichtc, 7ur Kriminalistik, zur Klimatologie sowie zu Waldschiden beschrieben werden. Der Optimimus iber die Zukunftsmöglichkeiten dieses Arbeitsgebietes wird an vielen Stellen verdeurlicht. Für alle !nteressierten bietet dieses Buch eine besonders umfassende Quelle von wertvollen Informationen

H. Schulz

Normenausschul Bauwesen im DIN (NABau): DIN-Normen und Norm-Entwürfe „Bauwesen“, 272 S. Berlin, Köln 1983: DIN Deutsches Institut für Normung e,V. Beuth Verlag. Bestellnr. II496. Brosch. 22,- DM.

Der NormenausschuB Bauwesen im DIN legt sein Verzeichnis der DIN-Normen und Normentwürfe 1983 vor. Dieses Verzeichnis wendet sich an alle, die mit dem Bauen zu tun haben, vor allem an die Baupraxis. aber auch an diejenigen, die Bauaufträge erteilen. Das Verzeichnis ist folgendernaßen gegliedert: 1. Bauwesen: Hier sind die Normen und Entwürfe aufgeführt, dic für Planung. Berechnung und
Konstruktion von baulichen Anlagen erforderlich sind. 2. Verdingungsordnung für Bauleistungen (VOB): In diesem Teil sind VOB Teile A. B und C zusammengefabt. Jeder ,Allgemeinen Technischen Vorschrifl" des Teiles $C$ der VOB sind die dort zitierten Normen und Entwürfe zugeordnet. 3. Standardleislungsbuch für das Bauwesen (StLB): Hier sind alle vorliegenden Leistungsbereiche mit Ausgabedatum und die in den Leistungsbereichen (LB) jewcils für Vertragstexte zitierten Normen und Entwürfe aufgeführt. 4. DIN-Taschenbücher: Diese Übersicht unterrichtet über dic Normen des Batuwesens, der Verdingungsordnung für Bauleistungen. und des Standardleistungsbuchs mit den in diesen Regelwerken zitierten Normen. Die Taschenbücher für Bauwesen und Bauleistungen enthalten unter dem jeweiligen Einzeltitel alle notwendigen z.Z. gültigen Normen. 5. Register: in cinem ausführlichen Register sind alle aufgeführten Normen und Norm-Entwürfe nach DIN-Nummer und Stichworten zu finden. Mit diesem Verzeichnis will der Normenausschuß Bauwesen einen Beitrag zur leichteren Handhabung und Awwendbarkeil des DIN-Normenwerkes im Bauwesen leisten. Das Verzeichnis enthäll deshalb atch DIN-Normen anderer Normenausschüsse.

Commonwealth Forestry Bureau: Machine Stress Grading and Nondestructive Testing of Wood. Annotated Bibliography No. F $29.46 \mathrm{~S}$ Slough 1982: Commonwealth Agricultural Bureau. Brosch. 10,15f

Verantworllich für die Zusammenstellung: Anne Handley, Comnonwealth Forestry Bureau, South Parks Road, Oxford OXI 3RD, U.K. Abgefragt wurden die Gebicte Forestry und Forest Products der CAB Datenbank, von 1973 bis 1982, sowic die Abstracts 1972 Die vorliegende Bibliographic stell eine Fortsetzung der früher veroffentlichten Annotated Bibliography No. F 7: "Machine StressGrading of Timber" dar, welche dic Literatur von 1961 bis 1971 umfaBte. Abgedruckt sind 194 Abstracts unter folgenden Headlines: General Methods, Particleboard, Glulam and Plywood, Joints, Standards and Grading Rules, Appendix (Literature Abstracted in 1972), Author Index.

K. A.Sorg 